Boise State University

ScholarWorks

9-28-2015

Enhanced Li Capacity in Functionalized Graphene: A First Principle Study with van der Waals Correction

\author{
Rajiv K. Chouhan \\ Boise State University \\ Pushpa Raghani \\ Boise State University
}




\title{
Enhanced Li capacity in functionalized graphene: A first principle study with van der Waals correction
}

\author{
Rajiv K. Chouhan a) and Pushpa Raghani \\ Department of Physics, Boise State University, 1910 University Dr., Boise, Idaho 83725, USA
}

(Received 25 March 2015; accepted 6 September 2015; published online 22 September 2015)

\begin{abstract}
We have investigated the adsorption of $\mathrm{Li}$ on graphene oxide using density functional theory. We show a novel and simple approach to achieve a positive lithiation potential on epoxy and hydroxyl functionalized graphene, compared to the negative lithiation potential that has been found on prestine graphene. We included the van der Waals correction into the calculation so as to get a better picture of weak interactions. A positive lithiation potential suggests a favorable adsorption of $\mathrm{Li}$ on graphene oxide sheets that can lead to an increase in the specific capacity, which in turn can be used as an anode material in Li-batteries. We find a high specific capacity of $\sim 860 \mathrm{mAhg}^{-1}$ by functionalizing the graphene sheet. This capacity is higher than the previously reported capacities that were achieved on graphene with high concentration of Stone-Wales $(75 \%)$ and divacancy $(16 \%)$ defects. Creating such high density of defects can make the entire system energetically unstable, whereas graphene oxide is a naturally occurring substance. (C) 2015 AIP Publishing LLC.

[http://dx.doi.org/10.1063/1.4931152]
\end{abstract}

\section{INTRODUCTION}

Energy storage is a major challenge in present times to meet the demand of consistent power supply, both in the portable devices like laptops, cameras, cellphone, etc., and in the bigger devices like electric vehicles, backup inverters, etc. Additionally, increase in the cost of fuel and in green house gases has given rise to the hybrid electric vehicle technology with a battery supplement, known as plugin hybrid electric vehicle (PHEV). ${ }^{1}$ For several years, PHEVs and many other appliances, such as lithium batteries, have been considered to be optimum candidates for rechargeable battery technology.

Although Li metal has a high theoretical capacity of $\sim 3842 \mathrm{mAhg}^{-1}$, rechargeable lithium batteries cannot use the bulk lithium metal due to safety issues, dendrite growth, rapid decay, and small recycling time..$^{2-6}$ To overcome these problems, many other materials and structure have been investigated, e.g., metal oxide such as $\mathrm{LiCoO}_{2}, \mathrm{LiFePO}_{4}$, and $\mathrm{LiMnO}_{2},{ }^{3-6}$ where $\mathrm{Li}$ atoms can be stored in layers within these metal oxides. However, these materials are found to have very small practical capacities of $140 \mathrm{mAhg}^{-1}, 170$ $\mathrm{mAhg}^{-1}$, and $119 \mathrm{mAhg}^{-1}$, respectively. Whereas graphite with theoretical capacity of $\sim 372 \mathrm{mAhg}^{-1}$ is used as conventional anode. Recently, significant work is being done to enhance the capacities of anodes as well as cathodes using the carbon based materials, ${ }^{7-13}$ like a composite anode based on carbon and silicon with a specific capacity of $\sim 1000 \mathrm{mAhg}^{-1}$ has been proposed. ${ }^{8}$ Similarly, a few more works ${ }^{7,13}$ also shows a hike in the capacity of the Li storage on graphene porous networks with Stone-Wales (SW) and divacancy (DV) defects. They reported an experimental capacity of 850 $\mathrm{mAhg}^{-1}$, whereas theoretical calculation predicts capacities of $698 \mathrm{mAhg}^{-1}$ and $590 \mathrm{mAhg}^{-1}$ with $75 \% \mathrm{SW}$ and $16 \%$

a)rajivchouhan@boisestate.edu
DV defects, respectively. Although even higher capacities with $100 \%$ SW and $25 \%$ DV defects are predicted from the calculation, creating graphene sheets with such a high percentage of defects is difficult and energetically unfavourable. For example, formation energies of SW defect is $\sim 5 \mathrm{eV},{ }^{14-16}$ and it is formed by rapid quenching from high temperature under the radiation of $90-100 \mathrm{eV}$ of electron energy. Similarly, formation energies of single- and divacancies in graphene sheet are $\sim 7.5 \mathrm{eV}$ and $\sim 8 \mathrm{eV}$, respectively. ${ }^{17-19}$ Although DV are thermodynamically more stable than single vacancy at high temperature, these vacancies are practically immobile. ${ }^{19}$ Additionally, removal of a large number of atoms can lead to the bending and wrapping of the sheet destroying its electronic properties. To overcome the problem, as well as taking the advantage of the fact that graphene sheet with nonstoichiometric nature can increase the lithiation potential (LP), we investigated the lithium adsorption over graphene oxide (GO). Interestingly, we find a positive LP on these functionalized graphene sheets suggesting a favorable adsorption of $\mathrm{Li}$ on $\mathrm{GO}$, leading to the specific capacity of $\sim 860 \mathrm{mAhg}^{-1}$. The most notable aspect of our proposed system is that it is simple and cost effective to prepare. Functionalized graphene can be created by simply treating it with strong acid/base ${ }^{20-24}$ that results in functional groups (epoxy (-O-) and hydroxyl (-OH)) attached to the sheet. For GO, one does not have to go through the reduction process of removing oxygen that naturally gets adsorbed on it or one does not have to create any high energy defects. Functionalized graphene shows a great potential for Li storage for making high capacity batteries. A specific capacity of $\sim 860 \mathrm{mAhg}^{-1}$ obtained by us is greater than that of $698 \mathrm{mAhg}^{-1}$ and $590 \mathrm{mAhg}^{-1}$ obtained with $75 \% \mathrm{SW}$ and $16 \% \mathrm{DV}$ of defects. Thus, GO is not only easy to prepare, it also gives very high specific capacities and could emerge as an ideal material for Li-batteries. 


\section{METHODS}

Density functional theory (DFT) calculations are performed in the pseudopotential formalism using Quantum Espresso $^{25}$ code. Due to the presence of lithium metal, a higher plane-wave cutoffs of $60 \mathrm{Ry}$ is used. Exchange interactions were treated within generalized gradient approximation with Perdew-Burke-Ernzerhof (PBE) ${ }^{26}$ functional form. We did include spin polarization in our calculations and found that there is a finite moment of $\sim 1 \mu_{\beta}$ in the presence of hydroxyl group but the magnetic moment disappears when $\mathrm{Li}$ is adsorbed close to functional groups. A maximum magnetic moment of $\sim 0.6 \mu_{\beta}$ is obtained in a few configurations with $\mathrm{Li}$ atom far away from the functional groups. As van der Waals (vdW) interactions play an important role in binding of Lithium ions over the graphene sheet ${ }^{27}$ we have included dispersion correction in our DFT calculations. ${ }^{28,29}$ We choose a computationally cost-effective "DFT-D2" method of Grimme, ${ }^{28}$ for vdW interactions. For $(1 \times 1)$ unit cell of graphene, we use a Monkhorst-pack k-mesh grid of $18 \times 18 \times 1$ and the corresponding k-mesh for bigger supercells. All the configurations have been relaxed with a force tolerance of $10^{-3} \mathrm{Ry} / \mathrm{bohr}$. To avoid interactions between images perpendicular to the plane of the sheet, a vacuum of $21 \AA$ is used. Using these parameters, we get C-C bond length of $1.42 \AA$ for pristine graphene that agrees well with the experiments. ${ }^{30}$ We calculate the adsorption energy (AE) and the lithiation potential as a function of Li concentration in pristine and functionalized graphene. LP can be determined from the Nernst equation using following equation:

$$
L P=\frac{\Delta G_{f}}{z F},
$$

where $z$ is charge on the $\mathrm{Li}$ ion and $\Delta G_{f}=\Delta E_{f}+T \Delta S_{f}-$ $P \Delta V_{f}$ is the change in Gibb's free energy. At room temperature, the entropic effects $\left(T \Delta S_{f}\right)$ and volume effects $\left(P \Delta V_{f}\right)$ will be very small compared ${ }^{31}$ to the formation energy $\Delta E_{f}$ and can be neglected. $\Delta E_{f}$ is defined as $\Delta E_{f}=-\left[E_{L i_{n}+\text { subs }}\right.$ $\left.-\left(n E_{L i}+E_{\text {subs }}\right)\right]$, where $E_{L i_{n}+\text { subs }}$ is the total energy of the lithiated GO, $E_{L i}$ is the total energy of a single lithium atom in body centred cubic (BCC) structure, and $E_{\text {subs }}$ is the total energy of substrate GO. On the other side, the $\mathrm{AE}$ of $\mathrm{Li}$ is calculated by using $E_{L i}$ for a gas phase Li atom instead of the metallic $\mathrm{Li}$ in the $\mathrm{BCC}$ structure.

\section{RESULTS AND DISCUSSION}

\section{A. Adsorption of Li on graphene}

We have looked at the adsorption of $\mathrm{Li}$ ion on graphene for various $\mathrm{Li}$ concentrations defined in terms of molar fraction $\mathrm{X}=\mathrm{A} /(\mathrm{A}+\mathrm{B})$, where $\mathrm{A}$ and $\mathrm{B}$ correspond to number of $\mathrm{Li}$ and carbon atoms in the supercell, respectively. Various $\mathrm{Li}$ concentrations $(\mathrm{X})$ are obtained either varying the cell size or keeping the cell size fixed and varying the number of $\mathrm{Li}$ atoms in the cell. Figure 1 shows the adsorption energies as a function of Li concentration from $1 \%$ to $10 \%$ by varying the cell size from $6 \times 6$ to $3 \times 3$, while the inset shows the variation of $\mathrm{Li}$ concentration from $11 \%$ to $33 \%$ by varying number of $\mathrm{Li}$ atoms in a fixed cell size of $2 \times 2$. First of all,

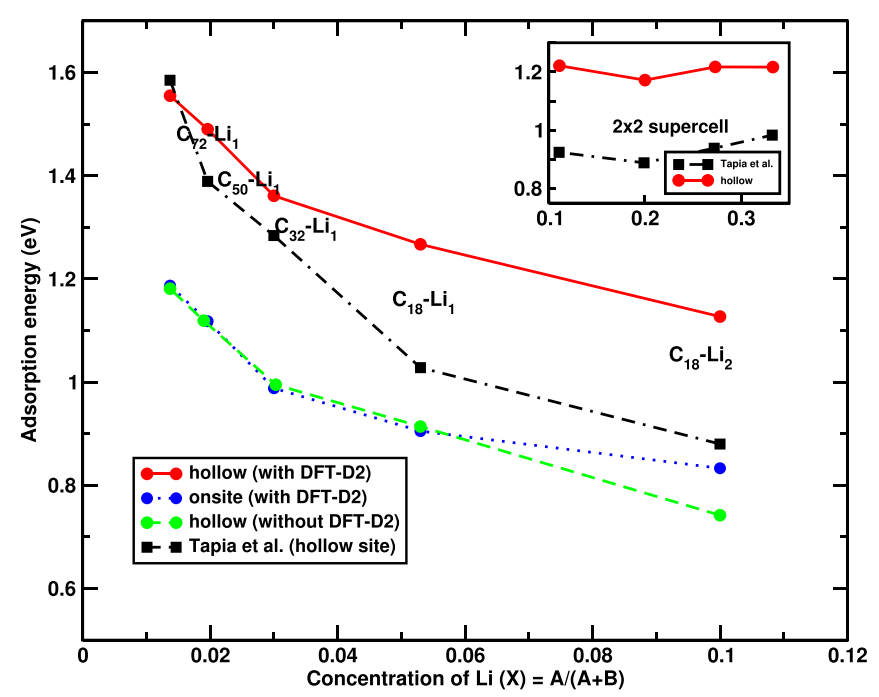

FIG. 1. Adsorption energies versus Li concentration from $1 \%$ to $10 \%$ on graphene by varying cell size for hollow and onsite positions. The inset shows adsorption energies versus Li concentrations from $11 \%$ to $33 \%$ in a fixed cell size of $2 \times 2$ at hollow site.

notice that $\mathrm{AE}$ of $\mathrm{Li}$ increases with decrease in $\mathrm{Li}$ concentration suggesting a repulsive interaction between $\mathrm{Li}$ atoms. $\mathrm{AE}$ at hollow site is the highest of all the other high symmetry sites (onsite, hollow, and bridge), in agreement with previous literature. $^{32,33} \mathrm{We}$ find that including vdW increases the adsorption of $\mathrm{Li}$ by $0.35 \mathrm{eV}$ irrespective of the $\mathrm{Li}$ concentration and the dispersion contribution to the $\mathrm{AE}$ comes out to be $0.48 \mathrm{eV}$, in good agreement with $0.45 \mathrm{eV}$ found by Allouche ${ }^{27}$ Even though the AE changes by a fixed amount of $\sim 0.35 \mathrm{eV}$ for all the $\mathrm{Li}$ concentrations, this difference is expected to change when we functionalize graphene with epoxy/hydroxyl groups, as these functional groups will change the charge distribution in the system. Hence, just by taking the vdW contribution separately will not give you the exact information about the AE and the proper inclusion of "DFTD2" inside the scf calculation is important.

\section{B. Lithiation of graphene oxide}

Here, we investigate GO as an anode material for $\mathrm{Li}^{-}$ batteries. It is well known that pristine graphene is not a suitable candidate for the $\mathrm{Li}$ adsorption due to its negative lithiation potential, ${ }^{7,34}$ and hence, it has a limitation to intercalate only between the spaces of graphene planes (with a theoretical capacity of $\sim 372 \mathrm{mAhg}^{-1}$ ). Recently, it has been shown that by introducing defects/vacancies, ${ }^{7,9,13}$ and functionalizing ${ }^{11,35}$ the graphene sheet, a positive LP can be achieved, and hence, one can enhance the battery capacity.

The structure of GO is still a puzzle due to its nonstoichiometric nature. Most recent NMR experiments ${ }^{36}$ suggest that the Carbon:Epoxy:Hydroxyl content on graphene can be varied by changing the environmental conditions. Commonly, the $\mathrm{C}: \mathrm{O}$ ratio in $\mathrm{GO}$ is found to be $2: 1$; however, after reduction process, this ratio can be increased to as high a value as $14.9: 1 .^{24,37-40}$ In our work, we use C:O ratio as 18:1 to 8:1. Under O-rich environment, the GO contains epoxy functional group (-O- attached to bridge site), while in H-rich environment, - $\mathrm{OH}$ group is formed (attached to 
carbon atom with orientation along hollow site) as shown in Figures 2(I) and 2(II), respectively. In general, there are numerous possibilities of basal functionalization through functional groups such as - $\mathrm{COOH},-\mathrm{O}-,-\mathrm{OH}$, and/or edge/ vacancy functionalization. In this paper, we have looked at the lithiation potential for three types of functionalized graphene: (I) epoxy covered, (II) hydroxyl covered, and (III) both, epoxy + hydroxyl covered as shown in Figure 2. These calculations are performed in a $3 \times 3$ supercell. Numbers in Figure 2 show the positions of $\mathrm{Li}$ atoms with respect to the functional group. Arrows in Figure 2(III) a-h indicate possible $-\mathrm{OH}$ positions and orientations with respect to the position of the epoxy group in same side as the epoxy group. In Figures 2(I) and 2(II), lithium is adsorbed on the same side, opposite side, and both sides of the functional group. However, for epoxy + hydroxyl (Figure 2(III)) functionalized graphene, $\mathrm{Li}$ is adsorbed only on the opposite side of the functional group.

For the case of single Li atom adsorption on the opposite side of epoxy group (site-2,3,4), we get the highest LP of $0.03 \mathrm{eV}$ at site-3 having specific capacity of $115 \mathrm{mAhg}^{-1}$. For the adsorption at site-5 and 7 on the same side as epoxy group, we get a LP of $1.44 \mathrm{eV}$ and $0.31 \mathrm{eV}$, respectively. This high LP of $1.44 \mathrm{eV}$ at site-5 is due to the binding of $\mathrm{Li}$ atom to the Oxygen atom causing it to break the double bond with the graphene sheet (see Figure 3(II)). A high LP of $1.66 \mathrm{eV}$ was also found by Stournara, ${ }^{11}$ for a similar configuration of Li bonded to the oxygen atom. This discrepancy of $0.22 \mathrm{eV}$ in LP could be due to the different cell sizes used in our calculations. At site-7, the Li atom remains at the hollow site and hence has a lower LP as expected. We also looked at adsorption of $\mathrm{Li}$ atom at site-2,4 on the same side as epoxy and found that in all two cases, Li atom gets bonded with the oxygen atom resulting in $1.44 \mathrm{eV}$ of LP (same as site-5 configuration Figure 3(II)), with O-Li bond getting oriented along the hollow site. Thus, LP gets greatly affected whether the $\mathrm{Li}$ atom is deposited on the same side or the opposite side of the epoxy group in the sheet. For the case of two $\mathrm{Li}$ atoms in cell at site- 5 and 7 and site- 2 and 5 placed along the side of epoxy, we obtain a LP of $1.11 \mathrm{eV}$ and $2.01 \mathrm{eV}$, respectively. For site- 5 and 7 one $\mathrm{Li}$ atom makes bond to oxygen and another Li atom remaining at site-7, while for site2 and $5 \mathrm{Li}$ atoms come near to oxygen with the position of oxygen at onsite position shown in Figure 3(III).

In the case hydroxyl functionalized graphene sheet, we get the highest LP of $0.86 \mathrm{eV}$ at site- 3 and 5 with Li atom deposited on the opposite side of the $-\mathrm{OH}$ group. Thus, LP in the hydroxyl functionalized graphene is higher than that of the epoxy functionalized graphene. With two and three $\mathrm{Li}$ atoms adsorbed on the opposite side, LP of $0.32 \mathrm{eV}$ and $0.22 \mathrm{eV}$ (specific capacity $230 \mathrm{mAhg}^{-1}$ and $345 \mathrm{mAhg}^{-1}$ ), respectively, are obtained. In the extreme case of fully covered (nine $\mathrm{Li}$ atoms per $-\mathrm{OH}$ group) $\mathrm{GO}$ a $\mathrm{LP}$ of $-0.20 \mathrm{eV}$ is found. However, when $\mathrm{Li}$ is placed on the same side as the $\mathrm{OH}$ group, it gets strongly bonded to the $-\mathrm{OH}$ group and the whole molecule $(\mathrm{Li}-\mathrm{OH})$ gets detached from graphene sheet. To investigate the dependence of LP on the functional group concentration, we used a $2 \times 2$ sized supercell with single epoxy/hydroxyl and Li adsorbed on the other side of the functional (i.e., C:O and $\mathrm{Li}: \mathrm{O}$ ratios of 8:1 and 4:1, respectively). With these concentrations, LP of $0.10 \mathrm{eV}$ and $0.01 \mathrm{eV}$ were obtained in presence of epoxy (956 $\mathrm{mAhg}^{-1}$ ) and hydroxyl $\left(948 \mathrm{mAhg}^{-1}\right)$, respectively [stars in Fig. 4]. This shows that the LP increases with the increase in functional group concentration.

We then investigated the LP when both groups (epoxy and $-\mathrm{OH}$ ) are presents in the graphene sheet and placed on the same side and $\mathrm{Li}$ in other side. The possible orientations

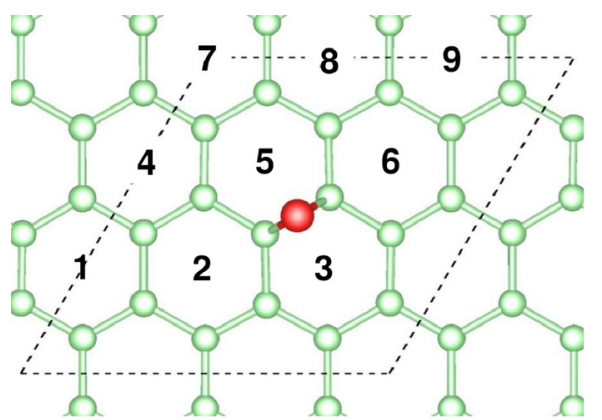

(I)

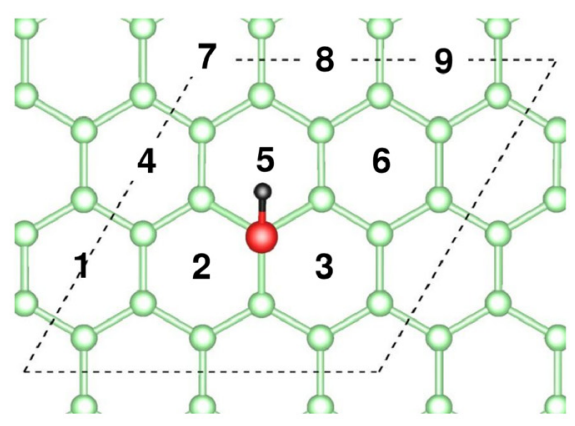

(II)
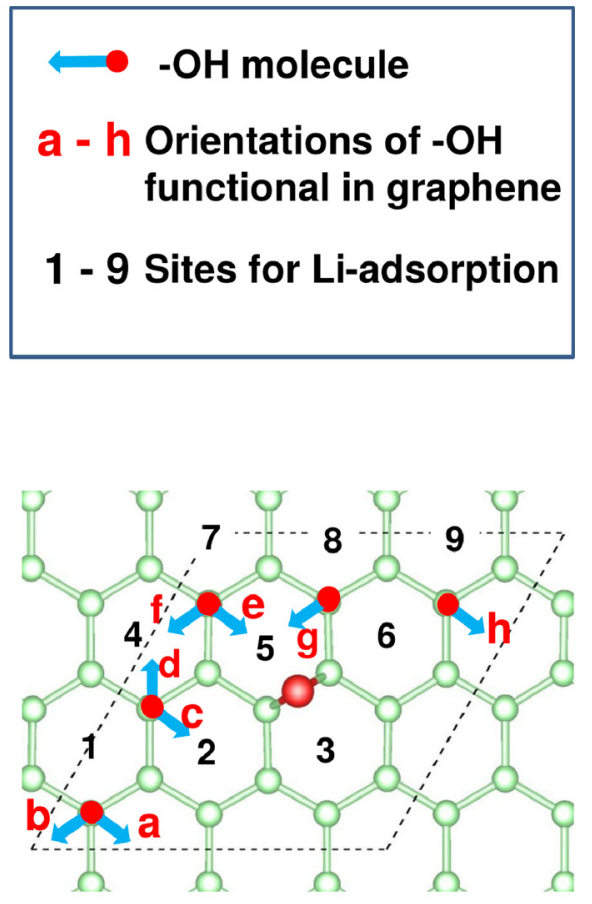

(III)
FIG. 2. Models of GO with three different functional groups: (I) Epoxy (II) Hydroxyl and (III) Epoxy + Hydroxyl, attached to graphene sheet in $3 \times 3$ supercell. Numbers show the adsorption sites of Li. In (III), blue arrows with red dots show the possible configurations (a-h) of hydroxyl radical with different orientations on the same side of epoxy functional. Color scheme of atomic spheres: $\mathrm{H}$ (black), $\mathrm{O}$ (red), and C (green). 


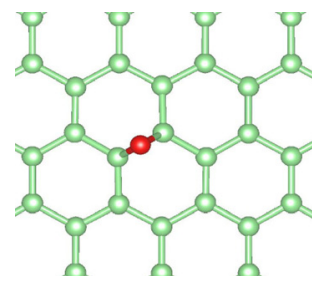

Top view

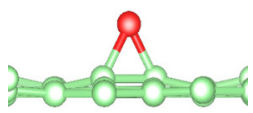

Side view

(I)

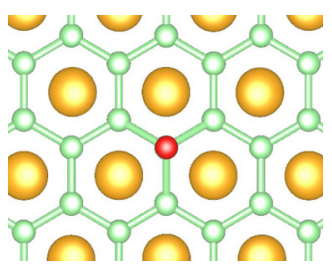

Top view

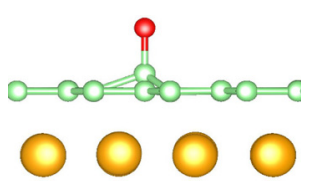

Side view (IV)

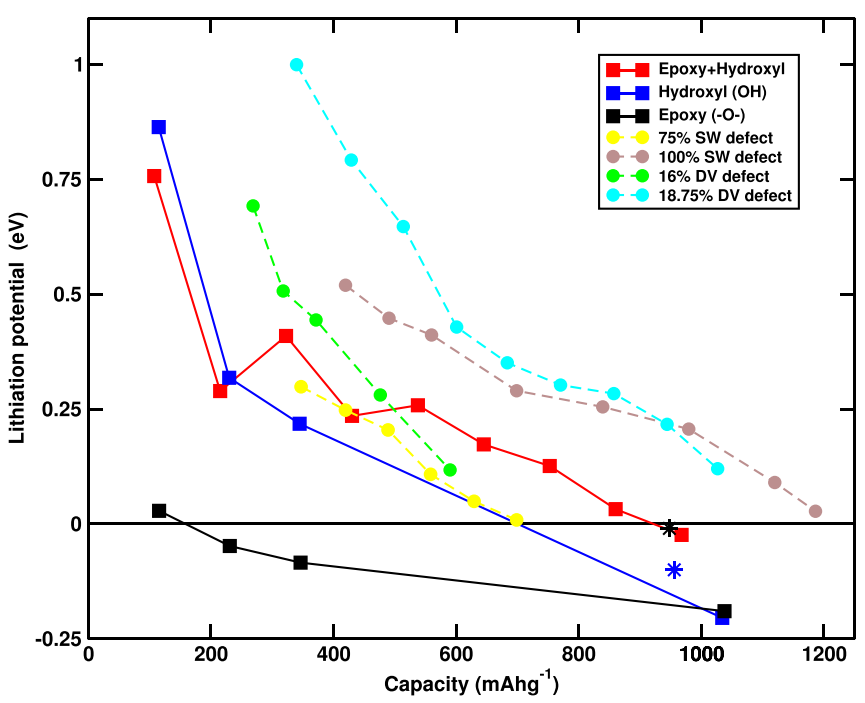

FIG. 4. Lithiation potential as a function of capacity for the cases of epoxy, hydroxyl, and epoxy + hydroxyl functionalized graphene. Squares and stars represent the LP from our data with C:O ratios of 18:1 and 8:1, respectively. $\mathrm{Li}$ is adsorbed on the opposite side of the functional group. Circles represent data from previous literature with SW and DV defects in graphene. ${ }^{7,13} \mathrm{~A}$ highest capacity of $\sim 860 \mathrm{mAhg}^{-1}$ is obtained on GO with epoxy + hydroxyl functionalization.
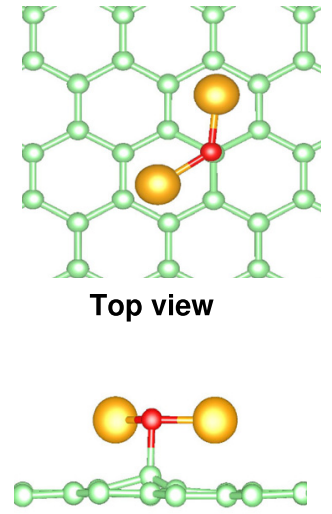

Side view

(III)

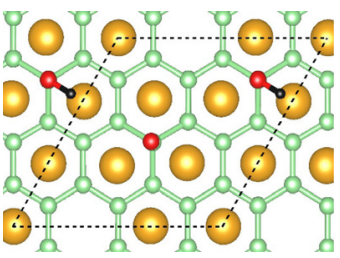

Top view

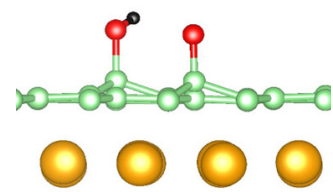

Side view

(VI)

of -OH with respect to epoxy are shown in Figure 2(III). Out of (a)-(h) configurations, the lowest energy configurations are found to be (e) and (h). Lithium atoms are placed on the opposite side of the functional groups. LP for one Li in $3 \times 3$ supercell $\left(107 \mathrm{mAhg}^{-1}\right)$ is $0.76 \mathrm{eV}$, which is $0.10 \mathrm{eV}$ less than that obtained with only - $\mathrm{OH}$ group. However, as we gradually increase the concentration of Li, LP increases compared to that with only $\mathrm{OH}$ group, as shown in Figure 4 (compare red squares with blue squares), e.g., LP at 315 $\mathrm{mAhg}^{-1}$ is $0.19 \mathrm{eV}$ greater than that with only $\mathrm{OH}$ group. The close analysis of the structure shows that, above 315 $\mathrm{mAhg}^{-1}$ capacities, epoxy group breaks one of its bond and oxygen gets attached to a single $\mathrm{C}$ atom at onsite position in the sheet. We observe a similar behaviour when epoxy contained graphene sheet was fully covered with $\mathrm{Li}$ on the opposite side (average $\mathrm{LP}=-0.19 \mathrm{eV}$ ) as shown in Figure 3(IV). This new behaviour of oxygen atom replicates a situation similar to that of the hydroxyl group that shows a higher LP compared to the epoxy group. This breaking of the bond of oxygen with one of the carbon atoms could be caused by the stretching of the graphene sheet in presence of the metallic Li layer on the opposite side. Charge redistribution in presence of Li atoms (particularly the 2 s orbital contribution) 
TABLE I. Lithiation potential with varying capacity for configurations (III)e and (III)h in Figure 2.

\begin{tabular}{lccccc}
\hline \hline & \multicolumn{2}{c}{ Configuration (III)e } & & \multicolumn{2}{c}{ Configuration (III)h } \\
\cline { 2 - 3 } \cline { 6 - 7 } Capacity $\left(\mathrm{mAhg}^{-1}\right)$ & LP $(\mathrm{eV})$ & sites & & LP $(\mathrm{eV})$ & sites \\
\hline 107 & 0.76 & $(5)$ & & 0.54 & $(6)$ \\
215 & 0.29 & $(4,5)$ & & 0.26 & $(6,9)$ \\
322 & 0.41 & $(4,5,7)$ & & 0.28 & $(4,6,9)$ \\
430 & 0.24 & $(3-5,7)$ & & 0.20 & $(4,6,7,9)$ \\
537 & 0.26 & $(2-5,7)$ & & 0.12 & $(2,3,5,6,9)$ \\
645 & 0.17 & $(2-7)$ & & 0.16 & $(2,4-7,9)$ \\
752 & 0.08 & $(2-8)$ & & 0.13 & $(2-7,9)$ \\
860 & 0.02 & $(2-9)$ & & 0.03 & $(1-7,9)$ \\
967 & -0.09 & $(1-9)$ & & -0.02 & $(1-9)$ \\
\hline \hline
\end{tabular}

and the singly bonded oxygen atom with graphene sheet at onsite position could be important factors for the boost in the LP with higher capacity. From Table I, notice that the configuration (III)e dominates the binding up to the capacity of $645 \mathrm{mAhg}^{-1}$ with LP of $0.17 \mathrm{eV}$, while from $752 \mathrm{mAhg}^{-1}$ capacity onwards configuration (III)h gives the highest capacity $\sim 860 \mathrm{mAhg}^{-1}$ with LP of $0.03 \mathrm{eV}$. Thus, we successfully achieve a positive LP up to the specific capacity of $\sim 860 \mathrm{mAhg}^{-1}$ just by placing the Lithium atoms only on one side of the sheet as shown in Figures 3(V) and 3(VI).

\section{CONCLUSIONS}

Lithium adsorption and lithiation potential on graphene oxide has been investigated using DFT. We find that epoxy and hydroxyl functionalized graphene sheet provides the highest specific capacity of $\sim 860 \mathrm{mAhg}^{-1}$. We have achieved higher capacities than previously reported capacities $^{13}$ of $698 \mathrm{mAhg}^{-1}$ and $590 \mathrm{mAhg}^{-1}$ with $75 \% \mathrm{SW}$ and $16 \%$ DV defects, respectively. Although even higher capacities of $1186 \mathrm{mAhg}^{-1}$ and $1029 \mathrm{mAhg}^{-1}$ were obtained by Dutta, ${ }^{13}$ with $100 \%$ SW and $18.75 \%$ (or higher) DV defects, respectively, we believe that creating such high density of defects would make the entire system energetically unstable leading to bending and wrapping of the $2 \mathrm{D}$ sheet. In this paper, we have shown the hike in Li storage capacity by attaching the functional group on one side of the sheet and $\mathrm{Li}$ on the other side. However, it would be possible to further increase the storage capacity by increasing the density of functional groups and $\mathrm{Li}$ atoms, i.e., by playing around with number of Li atoms per epoxy and/or per hydroxyl groups. Additionally, as both vacancy adsorption ${ }^{7,13}$ and functionalization (current work) show enhanced $\mathrm{Li}$ adsorption, functionalization and defects together can further increase the capacity and lithiation potential. To accurately predict the capacity of GO, one has to investigate the possibility of phase segregation ${ }^{41}$ in addition to the thermodynamic stability. In our future work, we are going to investigate the edge and interface effects on the Li capacity and LP.

We have also demonstrated that London's dispersion contribution plays an important role in binding of $\mathrm{Li}$ on graphene and GO sheets. Oxygen bonding and its position in GO plays an important role in raising the specific capacity of
GO. Thus, based on the first principle studies, we propose an efficient way to increase the capacity on a novel low-cost system (GO). Our results provide an insight to find a better anode material for rechargeable $\mathrm{Li}$ batteries without damaging or drastically modifying the properties of graphene.

\section{ACKNOWLEDGMENTS}

The authors thank the HPC center of Idaho National Laboratory for providing computational facilities. This work was financially supported from NSF CAREER award (DMR-1255584) and Research Corporation's Cottrell college Science award.

${ }^{1}$ D. Howell, T. Duong, J. B. Deppe, and I. Weinstock, Mater. Matters 3(4), 100 (2008); available at http://www.sigmaaldrich.com/content/dam/ sigma-aldrich/materials-science/material-matters/material_matters_v3n4.pdf.

${ }^{2}$ G.-A. Nazri and G. Pistoia, Lithium Batteries: Science and Technology (Springer, 2009), ISBN: 978-0-387-92675-9.

${ }^{3}$ K. Mizushima, P. C. Jones, P. J. Wiseman, and J. B. Goodenough, Mater. Res. Bull. 15, 783-789 (1980).

${ }^{4}$ J.-M. Tarascon and M. Armand, Nature 414, 359-367 (2001).

${ }^{5}$ R. Bhattacharyya, B. Key, H. Chen, A. S. Best, A. F. Hollenkamp, and C. P. Grey, Nat. Mater. 9, 504-510 (2010).

${ }^{6}$ F. Orsini, A. D. Pasquier, B. Beaudoin, J. M. Tarascon, M. Trentin, N. Langenhuizen, E. D. Beer, and P. Notten, J. Power Sources 76, 19-29 (1998).

${ }^{7}$ R. Mukherjee, A. V. Thomas, D. Datta, E. Singh, J. Li, O. Eksik, V. B. Shenoy, and N. Koratkar, Nat. Commun. 5, 3710 (2014).

${ }^{8}$ N. Liu, H. Wu, M. T. McDowell, Y. Yao, C. Wang, and Y. Cui, Nano Lett. 12, 3315-3321 (2012).

${ }^{9}$ X. Zhou, Y.-X. Yin, L.-J. Wan, and Y.-G. Guo, Chem. Commun. 48, 2198-2200 (2012).

${ }^{10}$ R. Krishnan, T.-M. Lu, and N. Koratkar, Nano Lett. 11, 377-384 (2011).

${ }^{11}$ M. E. Stournara and V. B. Shenoy, J. Power Sources 196, 5697-5703 (2011).

${ }^{12}$ V. Meunier, J. Kephart, C. Roland, and J. Bernholc, Phys. Rev. Lett. 88, 075506 (2002).

${ }^{13}$ D. Dutta, J. Li, N. Koratker, and V. B. Shenoy, Carbon 80, 305-310 (2014).

${ }^{14}$ L. Li, S. Reich, and J. Robertson, Phys. Rev. B 72, 184109 (2005).

${ }^{15}$ J. Ma, D. Alfe, A. Michaelides, and E. Wang, Phys. Rev. B 80, 033407 (2009).

${ }^{16}$ F. Banhart, J. Kotakoski, and A. V. Krasheninnikov, ACS Nano 5, 26-41 (2011).

${ }^{17}$ A. V. Krasheninnikov, P. O. Lehtinen, A. S. Foster, and R. M. Nieminen, Chem. Phys. Lett. 418, 132-136 (2006).

${ }^{18}$ J. Rossato, R. J. Baierle, A. Fazzio, and R. Mota, Nano Lett. 5, 197-200 (2005).

${ }^{19}$ A. A. El-Barbary, R. H. Telling, C. P. Ewels, M. I. Heggle, and P. R. Briddon, Phys. Rev. B 68, 144107 (2003).

${ }^{20}$ K. P. Loh, Q. Bao, G. Eda, and M. Chhowalla, Nat. Chem. 2, 1015 (2010).

${ }^{21}$ J. W. Suk, R. D. Piner, J. An, and R. S. Ruoff, ACS Nano 4, 6557-6564 (2010).

${ }^{22}$ W. S. Hummers and R. E. Offeman, J. Am. Chem. Soc. 80, 1339 (1958).

${ }^{23}$ D. C. Marcano, ACS Nano 4, 4806-4814 (2010).

${ }^{24}$ D. R. Dreyer, S. Park, C. W. Bielawski, and R. S. Ruoff, Chem. Soc. Rev. 39, 228-240 (2010).

${ }^{25}$ P. Giannozzi, J. Phys.: Condens. Matter 21, 395502 (2009).

${ }^{26}$ J. P. Perdew, K. Burke, and M. Ernzerhof, Phys. Rev. Lett. 77, 3865 (1996).

${ }^{27}$ A. Allouche and P. S. Krstic, Carbon 50, 510-517 (2012).

${ }^{28}$ S. Grimme, J. Comp. Chem. 27, 1787-1799 (2006).

${ }^{29}$ A. Tkatchenko and M. Scheffler, Phys. Rev. Lett. 102, 073005 (2009).

${ }^{30} \mathrm{P}$. Trucano and R. Chen, Nature 258, 136-137 (1975).

${ }^{31}$ M. K. Aydinol, A. F. Kohan, and G. Ceder, J. Power Sources 68, 664-668 (1997).

${ }^{32}$ K. T. Chan, J. B. Neaton, and M. L. Cohen, Phys. Rev. B 77, 235430 (2008).

${ }^{33}$ A. M. Garay-Tapia, A. H. Romero, and V. Barone, J. Chem. Theory Comput. 8, 1064-1071 (2012). 
${ }^{34}$ L.-J. Zhou, Z. F. Hou, and L.-M. Wu, J. Phys. Chem. C 116, 21780-21787 (2012).

${ }^{35}$ C. B. Robledo, M. Otero, G. Luque, O. Camara, D. Barraco, M. I. Rojas, and E. P. M. Leiva, Electrochim. Acta 140, 232-237 (2014).

${ }^{36}$ W. Cai, Science 321, 1815-1817 (2008).

${ }^{37}$ S. Zhou and A. Bongiorno, Nat. Sci. Rep. 3, 2484 (2013).
${ }^{38}$ G. Wang, J. Yang, J. Park, X. Gou, B. Wang, H. Liu, and J. Yao, J. Phys. Chem. C 112, 8192-8195 (2008).

${ }^{39}$ L. Staudenmaier, Ber. Dtsch. Chem. Ges. 31, 1481-1487 (1898).

${ }^{40}$ S. Stankovich, Carbon 45, 1558-1565 (2007).

${ }^{41}$ Y. Liu, Y. M. Wang, B. I. Yakobson, and B. C. Wood, Phys. Rev. Lett. 113, 028304 (2014). 